'Programa de Doctorado en Ciencias Humanas, Universidad de Talca, Talca, Chile.

2Programa de Magíster en Psicología Social, Universidad de Talca, Talca, Chile.

${ }^{3}$ Facultad de Psicología, Universidad de Talca, Talca, Chile. ${ }^{4}$ Programa de Vida Saludable, Actividad Física y Deportes, Universidad de Talca, Talca, Chile.

apsicólogo, estudiante del Programa de Doctorado en Ciencias Humanas de la Universidad de Talca.

bPsicólogo, estudiante del Programa de Magíster en Psicología Social de la Universidad de Talca.

'Psicólogo, Doctor en Psicología. dProfesora de Educación Física,

Magíster en Educación Física y Magíster en Ciencias de la Actividad Física.

eProfesor de Educación Física, Magíster en Ciencias de la Actividad Física.

Fuente de financiamiento: Proyecto de Desarrollo Integral Multidisciplinario en Colegios del Maule, Convenio de Desempeño en Humanidades, Artes y Ciencias Sociales (UTALCA-MINEDUC), Proyecto FONDECYT N ${ }^{\circ} 1161661$ y Beca CONICYT Doctorado Nacional. Los autores declaran no tener conflictos de interés.

Recibido el 16 de diciembre de 2019, aceptado el 1 de octubre de 2020.

Correspondencia a: Ismael Gallardo

Facultad de Psicología, Av. Lircay s/n, Talca, VII Región, Chile. igallardo@utalca.cl

\section{Escala de autoeficacia general: reevaluación de su evidencia de confiabilidad y validez en Chile}

\author{
MANUEL CLAVIJO ${ }^{1, \mathrm{a}}$, FELIPE YÉVENES ${ }^{2, \mathrm{~b}}$, \\ ISMAEL GALLARDO ${ }^{3, c}$, ANA MARÍA CONTRERAS ${ }^{4, \mathrm{~d}}$, \\ CRISTIAN SANTOS ${ }^{4, E}$
}

\section{The general self-efficacy scale (GSES): Reevaluation of its reliability and validity evidence in Chile}

Background: Self-efficacy is a psychological variable closely related to healthy behaviors. One of the most widely used instruments to measure this variable is the general self-efficacy scale (GSES). In Chile, the only psychometric study of this scale has important analytical limitations. Aim: To assess reliability and validity of GSES in a large Chilean sample. Material and Methods: The GSES and a self-esteem scale were applied to 2995 participants aged 11 to 76 years (60\% women). Results: The scale showed optimal levels of homogeneity and internal consistency. The exploratory factor analysis and the parallel analysis suggested a one-dimensional internal structure. However, this model showed a mediocre fit in the confirmatory factor analysis due to the residual correlation between a pair of items with high semantic similarity. A one-dimensional model specifying this residual correlation attained an acceptable fit in a new confirmatory factor analysis. The new model reached strict invariance according to sex, and partial strict invariance according to age. The latent factor of the GSES showed a positive association with the latent factor of a self-esteem scale. Conclusions: The GSES is an adequate scale to measure general self-efficacy in the Chilean population and allows unbiased comparisons based on sex or age.

(Rev Med Chile 2020; 148: 1452-1460)

Key words: Self Efficacy; Surveys and Questionnaires; Validation Study. 
adherencia a un tratamiento ${ }^{13}$, se comprenderían mejor desde la autoeficacia específica, y situaciones que demandan un repertorio de conductas de afrontamiento más variado y complejo, como un cuadro depresivo $^{14}$, se comprenderían mejor desde la autoeficacia general.

En el marco de la perspectiva general, uno de los instrumentos más utilizados es la Escala de Autoeficacia General (EAG) ${ }^{15}$. Con adaptaciones a más de 30 idiomas, se ha convertido, particularmente en ciencias de la salud, en una valiosa herramienta clínica e investigativa para determinar el potencial que tiene una persona para afrontar escenarios vitales con múltiples estresores, tales como la aparición de una enfermedad catastrófica $^{16}$, el padecimiento de afecciones crónicas $^{17}$ o cambios bruscos en las condiciones de vida ${ }^{18}$. Basada en una estructura interna unidimensional, la EAG ha mostrado adecuados niveles de confiabilidad, validez y funcionamiento de sus ítems en múltiples estudios que han analizado sus propiedades de medición ${ }^{19,20}$. En Chile, el único estudio psicométrico publicado data del año $2010^{21}$. Dicho trabajo, si bien aporta información relevante, presenta varias limitaciones analíticas. Específicamente, el análisis de confiabilidad se basó exclusivamente en el alfa de Cronbach y no en índices robustos a la cantidad de ítems como el omega de McDonald; la estructura interna se estimó mediante la interpretación subjetiva de la matriz de correlaciones y no con técnicas estadísticas que permiten determinar la dimensionalidad subyacente a los ítems, como el análisis factorial; la validez convergente se evaluó con la correlación entre las puntuaciones directas de la EAG y otras escalas de constructos teóricamente emparentados, y no con técnicas que aíslen la varianza no explicada por las variables latentes de estas escalas, como sí lo hacen los modelos de ecuaciones estructurales (MEE), y, por último, la muestra empleada fue pequeña $(n=360)$ considerando los estándares más exigentes ${ }^{22}$, lo que puede conducir a estimaciones imprecisas sobre las características psicométricas del instrumento.

En virtud de lo anterior, la presente investigación tiene como objetivo reevaluar la evidencia de confiabilidad y validez de la EAG en población chilena, en una muestra de gran tamaño, y empleando un set de técnicas analíticas que subsane las limitaciones del estudio nacional anterior.

\section{Material y Método}

\section{Muestra}

La muestra fue de 2995 participantes (mujeres $=60 \%$ ). El rango etario fue de 11 a 76 años $(M=24,31, D T=12,79$, mayores de edad $=52,3 \%)$. Los participantes fueron reclutados mediante un muestreo no probabilístico intencional en instituciones de educación primaria (alumnos y apoderados), secundaria (alumnos y apoderados) y universitaria (alumnos) de la Región del Maule, Chile. La aplicación fue en las instituciones.

\section{Instrumentos}

Escala de autoeficacia general (EAG): Se compone de 10 ítems (Tabla 1) que teóricamente responden a un único factor latente. En el estudio psicométrico chileno anterior se utilizó un formato de respuesta Likert de 4 puntos $(1=$ incorrecto,

Tabla 1. Ítems de la EAG

1. Puedo encontrar la forma de obtener lo que quiero aunque alguien se me oponga*

2. Puedo resolver problemas difíciles si me esfuerzo lo suficiente

3. Me es fácil persistir en lo que me he propuesto hasta llegar a alcanzar mis metas

4. Tengo confianza en que podría manejar eficazmente acontecimientos inesperados

5. Gracias a mis propias cualidades y recursos puedo superar situaciones imprevistas**

6. Cuando me encuentro en dificultades puedo permanecer tranquilo/a porque cuento con las habilidades necesarias para manejarlas

7. No importa el problema, porque por lo general soy capaz de manejarlo***

8. Puedo resolver la mayoría de los problemas si me esfuerzo lo necesario

9. Si me encuentro en una situación difícil, generalmente se me ocurre qué debo hacer

10. Al tener que hacer frente a un problema, generalmente se me ocurren varias alternativas de cómo resolverlo

Nota: *En la versión chilena anterior, este ítem decía "Puedo encontrar la manera de obtener lo que quiero, aunque alguien se me oponga"; **En la versión chilena anterior, este ítem decía "Gracias a mis cualidades y recursos puedo superar situaciones imprevistas"; ***En la versión chilena anterior, este ítem decía "Venga lo que venga, por lo general soy capaz de manejarlo". 
$2=$ apenas cierto, $3=$ más bien cierto, $4=$ cierto), no obstante, en el presente estudio se optó por un formato de 7 puntos distinto $(1=$ completamente en desacuerdo y $7=$ completamente de acuerdo). Esta determinación obedeció a dos razones: suele tener un uso más común en el contexto nacional $\mathrm{y}$ ofrece un punto medio que permite una valoración neutral ${ }^{23}$. Adicionalmente, se efectuaron pequeñas modificaciones de redacción en tres ítems de la versión antigua, con el objetivo de lograr una mejor comprensión de estos (Tabla 1). Como todos los ítems tienen la misma dirección, ninguno fue recodificado en los análisis.

Escala de autoestima de Rosenberg abreviada (EARA): Se utilizaron tres ítems (Tabla 2), pertenecientes al factor positivo descrito en el estudio psicométrico chileno de esta escala ${ }^{24}$. El formato de respuesta fue el mismo Likert de siete puntos de la EAG. Como todos los ítems tienen la misma dirección, ninguno fue recodificado en los análisis.

\section{Tabla 2. Ítems de la EARA}

1. Siento que soy una persona digna de aprecio, al menos en igual medida que las demás

2. Tengo una actitud positiva hacia mí mismo/a

3. En general, estoy satisfecho conmigo mismo/a

\section{Análisis de datos}

La muestra total fue dividida aleatoriamente en dos submuestras para evitar la capitalización del $\operatorname{azar}^{25}$. La submuestra 1 quedó conformada por 1.497 participantes (mujeres $=60,6 \%$ ) y un rango etario de 11 a 76 años $(M=24,28$, $\mathrm{DT}=12,87$, mayores de edad $=52,3 \%$ ). La submuestra 2 quedó conformada por 1498 participantes (mujeres $=59,5 \%$ ) y un rango etario de 11 a 74 años $(\mathrm{M}=24,34, \mathrm{DT}=12,71$, mayores de edad $=52,4 \%)$.

En la submuestra 1 se realizó el análisis de los ítems (media, desviación típica, asimetría, curtosis, correlación ítem-total corregida) y confiabilidad (alpha de Cronbach y omega de McDonald), así como también el análisis factorial exploratorio (AFE) de la EAG. La adecuación de los datos para el AFE se evaluó con la prueba de esfericidad de Bartlett y la medida Kaiser-Meyer-Olkin (KMO).
Para el AFE se usó el método de estimación mínimos cuadrados no ponderados (ULS), que permite factorizar sin necesidad de supuestos distribucionales $^{26}$. El número de factores se determinó mediante el análisis paralelo de Horn e índices de ajuste como el índice Gamma (GFI) y el error cuadrático medio de aproximación (RMSEA). Los programas estadísticos usados fueron SPSS $21 \mathrm{y}$ FACTOR 10.9.02.

En la submuestra 2 se realizó el análisis factorial confirmatorio (AFC), la evaluación de invarianza de medida según sexo y edad, y una evaluación de la validez convergente de la EAG. En todos estos análisis se usó el método de estimación máxima verosimilitud robusto (MLR), que es idóneo cuando los supuestos de normalidad no son estrictamente cumplidos ${ }^{27}$. En el AFC, siguiendo las recomendaciones de la literatura especializada ${ }^{28,29}$, se usó un conjunto de índices de ajuste del modelo. Estos fueron el chi-cuadrado $\left(\chi^{2}\right)$, el índice de ajuste comparativo (CFI), el RMSEA y la raíz cuadrática media residual estandarizada (SRMR). Para evaluar la invarianza de medida se realizó un AFC multigrupo con la siguiente secuencia de $\left.\operatorname{pasos}^{28}: 1\right)$ se evaluó el modelo en cada grupo por separado (en sexo: hombres/mujeres; en edad: menores de edad/mayores de edad); luego, 2) se evaluó la equivalencia estructural del modelo entre los grupos simultáneamente (modelo con invarianza configuracional); a continuación, 3) al modelo con invarianza configuracional se le añadió la restricción de que las cargas factoriales fueran equivalentes entre los grupos (modelo con invarianza métrica); en seguida, 4) al modelo con invarianza métrica se le añadió la restricción de que los interceptos fueran equivalentes entre los grupos (modelo con invarianza escalar); y por último, 5) al modelo con invarianza escalar se le añadió la restricción de que los residuos fueran equivalentes entre los grupos (modelo con invarianza estricta). Los pasos 1 y 2 se evaluaron con los índices de ajuste del AFC, mientras que los pasos restantes se evaluaron usando la diferencia tanto en CFI como en RMSEA entre el modelo con la restricción añadida y su precedente (e.g., CFI del modelo con invarianza escalar menos CFI del modelo con invarianza métrica). La invarianza es lograda cuando la diferencia entre modelos contrastados es menor que 0,01 en CFI y 0,015 en RMSEA $^{30}$. Para recoger evidencia de validez convergente, se evaluó la correlación entre las 
Reevaluación de la escala de autoeficacia general en Chile - M. Clavijo et al

variables latentes de la EAG y la EARA, mediante un MEE. Se escogió esta última escala porque su constructo a la base (autoestima) ha mostrado una asociación positiva con la autoeficacia general ${ }^{21}$. El programa estadístico usado fue Mplus 7. La Tabla 3 sintetiza los indicadores y el criterio de idoneidad usado.

\section{Consideraciones éticas}

Previo a responder los cuestionarios, los participantes mayores y menores de edad firmaron un documento de consentimiento y asentimiento informado, respectivamente. En el caso de los menores de edad, los tutores legales aceptaron su participación mediante la firma de un documento de consentimiento y autorización. Los protocolos fueron revisados por el comité de ética de la Universidad de Talca.

\section{Resultados}

Análisis de los ítems y confiabilidad de la EAG

La Tabla 4 muestra el análisis de los ítems de la EAG en la submuestra 1. Todos los ítems tuvieron una media mayor que el punto medio de

Tabla 3. Indicadores usados en los análisis y su criterio de idoneidad

\begin{tabular}{|c|c|}
\hline Indicador & Criterio \\
\hline Alfa de Cronbach ${ }^{31}$ & $>0,8$ \\
\hline Omega de McDonald 31 & $>0,8$ \\
\hline Prueba de esfericidad de Bartlett ${ }^{32}$ & $p<0,05$ \\
\hline $\mathrm{KMO}^{32}$ & $\geq 0,6$ \\
\hline Análisis paralelo de Horn 22 & Factores deben tener autovalores mayores a los obtenidos por azar \\
\hline $\mathrm{GFI}^{33}$ & $>0,9$ \\
\hline RMSEA 33 & $<0,08$ \\
\hline$\chi^{228}$ & $p>0,05$ \\
\hline $\mathrm{CFI}^{33}$ & $>0,9$ \\
\hline $\mathrm{SRMR}^{34}$ & $<0,08$ \\
\hline
\end{tabular}

Tabla 4. Análisis y carga factorial de los ítems de la EAG

\begin{tabular}{|ccccccc|}
\hline Ítem & Media & $\begin{array}{c}\text { Desviación } \\
\text { típica }\end{array}$ & Asimetría & Curtosis & $\begin{array}{c}\text { r ítem-total } \\
\text { corregida }\end{array}$ & $\begin{array}{c}\text { Carga } \\
\text { factorial }\end{array}$ \\
\hline 1 & 4,867 & 1,828 & $-0,713$ & $-0,399$ & 0,367 & 0,385 \\
\hline 2 & 6,156 & 1,135 & $-1,841$ & 4,179 & 0,584 & 0,619 \\
\hline 3 & 5,657 & 1,355 & $-1,190$ & 1,375 & 0,588 & 0,622 \\
\hline 4 & 5,545 & 1,329 & $-0,999$ & 0,943 & 0,700 & 0,744 \\
\hline 5 & 5,509 & 1,326 & $-1,096$ & 1,371 & 0,699 & 0,747 \\
\hline 6 & 5,277 & 1,401 & $-0,863$ & 0,658 & 0,643 & 0,694 \\
\hline 7 & 5,124 & 1,433 & $-0,771$ & 0,425 & 0,689 & 0,739 \\
\hline 8 & 5,805 & 1,233 & $-1,297$ & 2,008 & 0,692 & 0,746 \\
\hline 9 & 5,580 & 1,289 & $-1,173$ & 1,683 & 0,676 & 0,731 \\
\hline 10 & 5,487 & 1,329 & $-0,955$ & 0,889 & 0,626 & 0,681 \\
\hline
\end{tabular}

Nota: $r=$ correlación. 
la escala y la desviación típica fue similar en cada uno de ellos. La asimetría y curtosis, exceptuando en los ítems 2 y 8 , se ubicaron dentro del rango -2 y 2 , lo cual refleja una distribución cercana a la normalidad $^{29}$. La correlación ítem-total corregida da cuenta de una adecuada homogeneidad de los ítems, puntuando sobre el criterio mínimo de $0,3^{32}$. En cuanto a la confiabilidad de la EAG, tanto el coeficiente alfa de Cronbach $(\alpha=0,883)$ como el coeficiente omega de McDonald $(\omega=0.893)$ mostraron niveles satisfactorios.

\section{Análisis factorial exploratorio}

Antes de iniciar el AFE en la submuestra 1 se evaluó la adecuación de los datos. Las pruebas de esfericidad de Bartlett $\left(\chi_{45}^{2}=6717,900\right.$; $\mathrm{p}<0,001)$ y Kaiser-Meyer-Olkin $(\mathrm{KMO}=0,917)$ confirmaron este requisito preliminar. El AFE ejecutado mediante el método de estimación ULS, coincidentemente con el modelo teórico, arrojó una solución de un único factor latente. La varianza total explicada fue de $51,21 \%$ y todas las cargas factoriales de los ítems estuvieron sobre 0,3 (Tabla 4). Adicionalmente, el análisis paralelo (Figura 1) y los indicadores de ajuste $(\mathrm{GFI}=0,991$;
RMSEA $=0,070)$ mostraron resultados consistentes con la unidimensionalidad sugerida.

\section{Análisis factorial confirmatorio}

Al realizar el AFC en la submuestra 2, el modelo unidimensional (1D) logró un ajuste bajo los niveles aceptables en la mayoría de los índices (Tabla 5). Para encontrar las causas de este problema se revisaron los índices de modificación del modelo, revelándose que los residuos de los ítems 9 y 10 tenían una correlación considerable. No obstante, este problema ya ha sido reportado en la literatura de la escala y su explicación recae en la redacción excesivamente parecida que tiene este par de ítems ${ }^{35}$. Dicha redundancia hace que estos compartan más varianza que aquella que es directamente explicada por el factor común, lo que deteriora el ajuste del modelo al no estar especificado en el mismo. Por este motivo se probó un modelo unidimensional modificado (1DM) que especificó dicha correlación residual. Como se puede observar en la Tabla 5, basado en los índices CFI, RMSEA y SRMR, este nuevo modelo logró un ajuste aceptable. Si bien el $\chi^{2}$ se mantuvo fuera del criterio de idoneidad, esto fue

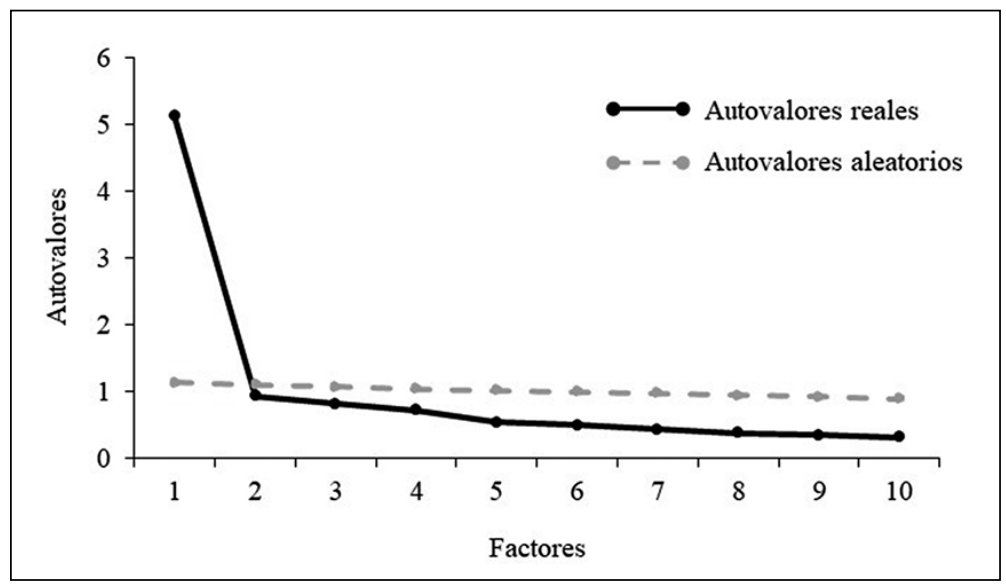

Figura 1. Gráfico del análisis paralelo de Horn. El número de matrices de correlación aleatorias empleado en el análisis fue de 500 .

Tabla 5. Índices de ajuste de los modelos

\begin{tabular}{|ccccccc|}
\hline Modelo & $\boldsymbol{\chi}^{\mathbf{2}}$ & gl & CFI & RMSEA (IC 90\%) & SRMR \\
\hline 1D & $389,048^{*}$ & 35 & 0,886 & $0,082(0,075-0,090)$ & 0,048 \\
\hline 1DM & $287,459^{*}$ & 34 & 0,918 & $0,071(0,063-0,078)$ & 0,041 \\
\hline
\end{tabular}

Nota: ${ }^{*} \mathrm{p}<0,001, \mathrm{gl}=$ grados de libertad, $\mathrm{IC}=$ intervalo de confianza. 
Tabla 6. Pruebas de invarianza del modelo 1DM según sexo y edad

\begin{tabular}{|c|c|c|c|c|c|c|c|}
\hline Variable & $\chi^{2}$ & gl & CFI & RMSEA (IC 90\%) & SRMR & $\Delta C F I$ & DRMSEA \\
\hline \multicolumn{8}{|l|}{ Sexo } \\
\hline Hombres & $106,599 *$ & 34 & 0,936 & $0,061(0,048-0,074)$ & 0,041 & & \\
\hline Mujeres & $198,087^{*}$ & 34 & 0,910 & $0,075(0,065-0,086)$ & 0,043 & & \\
\hline Configuracional & 308,361 * & 68 & 0,920 & $0,070(0,062-0,078)$ & 0,042 & & \\
\hline Métrica & $324,480 *$ & 77 & 0,918 & $0,067(0,060-0,075)$ & 0,053 & $-0,002$ & $-0,003$ \\
\hline Escalar & $357,423^{*}$ & 86 & 0,910 & $0,066(0,059-0,074)$ & 0,056 & $-0,008$ & $-0,001$ \\
\hline Estricta & $355,170^{*}$ & 96 & 0,914 & $0,061(0,055-0,068)$ & 0,074 & 0,004 & $-0,005$ \\
\hline \multicolumn{8}{|l|}{ Edad } \\
\hline$<18$ & $161,378^{*}$ & 34 & 0,909 & $0,076(0,064-0,088)$ & 0,046 & & \\
\hline$>18$ & $123,719^{*}$ & 34 & 0,935 & $0,061(0,050-0,073)$ & 0,039 & & \\
\hline Configuracional & $280,824^{*}$ & 68 & 0,923 & $0,068(0,060-0,076)$ & 0,043 & & \\
\hline Métrica & $299,176^{*}$ & 77 & 0,920 & $0,065(0,057-0,073)$ & 0,056 & $-0,003$ & $-0,003$ \\
\hline Escalar & $326,562 *$ & 86 & 0,913 & $0,064(0,057-0,072)$ & 0,058 & $-0,007$ & $-0,001$ \\
\hline Estricta & $493,962 *$ & 96 & 0,856 & $0,078(0,071-0,085)$ & 0,164 & $-0,057$ & 0,014 \\
\hline Estricta (pa) & $339,904^{*}$ & 91 & 0,910 & $0,063(0,056-0,071)$ & 0,067 & $-0,003$ & $-0,001$ \\
\hline
\end{tabular}

Nota: ${ }^{*} \mathrm{p}<0,001, \Delta=$ diferencia, $<18=$ menores de edad, $>18=$ mayores de edad, $\mathrm{pa}=$ parcial.

predecible por ser susceptible a verse afectado en muestras grandes ${ }^{28}$.

\section{Invarianza de medida}

El modelo 1DM fue sometido a una evaluación de invarianza según sexo y edad mediante un AFC multigrupo en la submuestra 2 (Tabla 6). En cuanto a la variable sexo, el modelo superó todas las pruebas de rigor, logrando un ajuste aceptable en el grupo de hombres y mujeres de manera independiente, y equivalencia intergrupo en términos de estructura factorial (invarianza configuracional), cargas factoriales (invarianza métrica), interceptos (invarianza escalar) y residuos (invarianza estricta). En cuanto a la variable edad, el modelo tuvo el mismo comportamiento, salvo en la invarianza estricta. En concreto, cuando se impuso la restricción de equivalencia intergrupo en los residuos, el ajuste experimentó un deterioro sustancial. La inspección de los índices de modificación sugirió liberar de esta restricción a los ítems $2,6,8,9$ y 10 , lo cual, una vez sometido a prueba, permitió alcanzar una invarianza estricta parcial.

\section{Evidencia de validez convergente}

Mediante un MEE se evaluó la correlación entre las variables latentes de la EAG y la EARA en la submuestra 2. El modelo tuvo un ajuste aceptable $\left(\chi^{2}=385,790, \mathrm{gl}=63, \mathrm{p}<0,001\right.$;
$\mathrm{CFI}=0,925$; RMSEA $=0,059$ [90\% IC 0,053$0,064], \mathrm{SRMR}=0,043)$ y la correlación, tal como se esperaba, fue positiva y significativa $(r=0,572$, $\mathrm{p}<0,001)$. El MEE se muestra en la Figura 2.

\section{Discusión}

El objetivo de este estudio fue reevaluar la evidencia de confiabilidad y validez de la EAG en población chilena, empleando un set de técnicas analíticas que atiende las limitaciones del estudio psicométrico nacional anterior.

El análisis de los ítems y confiabilidad de la escala mostró óptimos niveles de homogeneidad y consistencia interna, lo cual coincide con los hallazgos encontrados en el contexto nacional ${ }^{21} \mathrm{e}$ internacional ${ }^{19,35,36}$.

Respecto a la estructura interna, el AFE sugirió el mismo modelo unidimensional que ha sido propuesto en la literatura especializada ${ }^{19}$, el cual fue refrendado, posteriormente, por el AFC, aunque con la especificación de una correlación residual entre los ítems 9 y 10 para lograr un ajuste aceptable. Esta especificación, ya reportada en un estudio reciente ${ }^{35}$, se explica por la redacción excesivamente similar que posee este par de ítems, lo que los hace compartir más varianza de la explicada por la estructura unidimensional latente. 


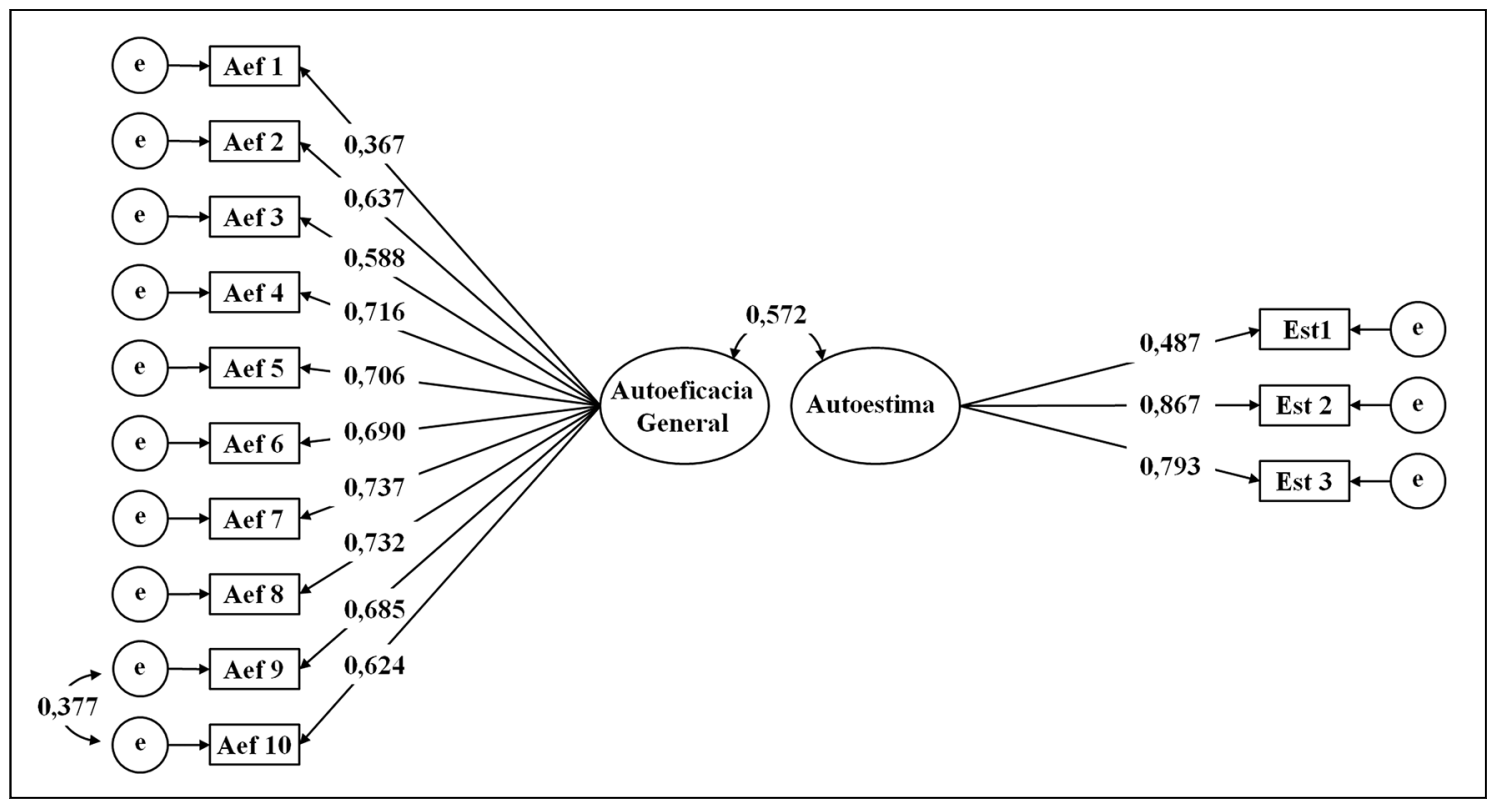

Figura 2. Gráfico de la correlación entre las variables latentes de la EAG y EARA en un MEE. Las elipses representan las variables latentes, los rectángulos los ítems, los círculos las varianzas residuales, las flechas rectas unidireccionales una relación causal y las flechas curvas bidireccionales una correlación. Los números sobre las flechas rectas que nacen desde las variables latentes son el valor de las cargas factoriales, mientras que los números sobre las flechas curvas son el valor de las correlaciones. Todos los parámetros fueron significativos. Todas las cargas factoriales están estandarizadas.

Lo anterior no implica problema en el uso de la EAG en contextos clínicos o aplicados, ya que sólo sugiere redundancia semántica en ese par de ítems. En este sentido, como la unidimensionalidad no está afectada, el uso del puntaje promedio como indicador de autoeficacia general no tiene inconvenientes. No obstante, en el caso de los contextos investigativos, esta especificación deberá considerarse en los análisis de variables latentes para así evitar problemas de ajuste al momento de modelar los datos. En cuanto a la generalidad del modelo, el AFC multigrupo apoyó la obtención de invarianza hasta el nivel estricto en la variable sexo, lo que permite que las puntuaciones medias de hombres y mujeres sean comparables sin sesgos de medida significativos. En el caso de la variable edad, la invarianza se alcanzó a un nivel estricto de manera parcial, lo cual, en sí mismo, tampoco es problemático porque los residuos son ajenos al objeto medido ${ }^{28}$, que es el factor latente (modelo, cargas e interceptos mantienen equivalencia intergrupal). En este sentido, la comparación entre grupos de mayores y menores de edad sigue siendo factible, tal como en el caso del sexo.
En lo concerniente a la evidencia de validez convergente, el MEE mostró una asociación positiva entre los factores latentes de la EAG y la EARA, lo cual es consistente con la teoría a la base y hallazgos previos ${ }^{21,37}$.

En síntesis, la EAG cumple con las condiciones psicométricas para ser utilizada en población chilena, ya sea con propósitos clínicos, aplicados o investigativos, y es idónea para hacer comparaciones no sesgadas según sexo o edad.

Por último, próximos estudios deberán contribuir a superar limitaciones del presente estudio, como la obtención de un muestreo más representativo de la heterogeneidad nacional (distintas regiones), el examen de evidencias de validez adicionales (discriminante y de criterio), o el análisis del funcionamiento diferencial de los ítems.

\section{Referencias}

1. Bandura A. Self-efficacy: Toward a unifying theory of behavioral change. Psychol Rev 1977; 84 (2): 191-215.

2. Bandura A. Self-efficacy. En: Ramachaudran VS, Editor, 
Encyclopedia of human behavior. New York, US: Editorial Academic Press; 1994. p. 71-81.

3. Bandura A. Social cognitive theory: An agentic perspective. Annu Rev Psychol 2001; 52 (1): 1-26.

4. Ammassari A, Trotta MP, Murri R, et al. Correlates and predictors of adherence to highly active antiretroviral therapy: overview of published literature. J Acquir Immune Defic Syndr 2002; 31 (3): 123-7.

5. Schwarzer R, Renner B. Social-cognitive predictors of health behavior: Action self-efficacy and coping self-efficacy. Health Psychol 2000; 19 (5): 487-95.

6. Kelly JF, Magill M, Stout RL. How do people recover from alcohol dependence? A systematic review of the research on mechanisms of behavior change in Alcoholics Anonymous. Addict Res Theory 2009; 17 (3): 236-59.

7. Bauman AE, Reis RS, Sallis JF, Wells JC, Loos RJ, Martin BW. Correlates of physical activity: why are some people physically active and others not? Lancet 2012; 380 (9838): 258-71.

8. Wingood GM, DiClemente RJ, Mikhail I, Lang DL, McCree DH, Davies SL, et al. A randomized controlled trial to reduce HIV transmission risk behaviors and sexually transmitted diseases among women living with HIV: The WiLLOW Program. J Acquir Immune Defic Syndr 2004; 37 (2): 58-67.

9. Hunkeler M, Katon W, Tang L, Williams J, Kroenke K, Lin E, et al. Long term outcomes from the IMPACT randomised trial for depressed elderly patients in primary care. BMJ 2006; 332 (7536): 259-63.

10. Schwarzer R. Modeling health behavior change: How to predict and modify the adoption and maintenance of health behaviors. Appl Psychol 2008; 57 (1): 1-29.

11. Shelton SH. Developing the construct of general self-efficacy. Psychological Reports 1990; 66 (3): 987-94.

12. Schwarzer R, Bäßler J, Kwiatek P, Schröder K, Zhang JX. The assessment of optimistic self-beliefs: Comparison of the German, Spanish, and Chinese versions of the general self-efficacy scale. Appl Psychol 1997; 46 (1): 69-88.

13. Johnson MO, Neilands TB, Dilworth SE, Morin SF, Remien RH, Chesney MA. The role of self-efficacy in HIV treatment adherence: Validation of the HIV treatment adherence self-efficacy scale (HIV-ASES). J Behav Med 2007; 30 (5): 359-70.

14. Volz M, Möbus J, Letsch C, Werheid K. The influence of early depressive symptoms, social support and decreasing self-efficacy on depression 6 months post-stroke. J Affect Disord 2016; 206: 252-5.

15. Schwarzer R, Jerusalem M. Generalized Self-Efficacy Scale. En: Weinman J, Wright S, Johnston M, Editores, Measures in health psychology: A user's portfolio. Causal and control beliefs. Windsor, UK: editorial
NFER-Nelson; 1995. p. 35-7.

16. Haugland T, Wahl AK, Hofoss D, DeVon HA. Association between general self-efficacy, social support, cancer-related stress and physical health-related quality of life: a path model study in patients with neuroendocrine tumors. Health Qual Life Outcomes 2016; 14: 1-7.

17. Cramm JM, Strating MMH, Roebroeck ME, Nieboer AP. The Importance of General Self-Efficacy for the Quality of Life of Adolescents with Chronic Conditions. Soc Indic Res 2013; 113: 551-61.

18. Brink E, Alsén P, Herlitz J, Kjellgren K, Cliffordson C. General self-efficacy and health-related quality of life after myocardial infarction. Psychol Health Med 2012; 17(3): 346-55.

19. Scholz U, Dona BG, Sud S, Schwarzer R. Is general self-efficacy a universal construct? Psychometric findings from 25 countries. Eur J Psychol Assess 2002; 18 (3): 242-51.

20. Lönnfjord V, Hagquist C. The psychometric properties of the Swedish version of the general self-efficacy scale: A Rasch analysis based on adolescent data. Curr Psychol 2017; 37 (4): 703-15.

21. Cid P, Orellana A, Barriga O. Validación de la escala de autoeficacia general en Chile. Rev Med Chil 2010; 138 (5): 551-7.

22. Lloret-Segura S, Ferreres-Traver A, Hernández-Baeza A, Tomás-Marco I. El análisis factorial exploratorio de los ítems: una guía práctica, revisada y actualizada. Anales de Psicología / Annals of Psychology 2014; 30 (3): 115169.

23. DeCastellarnau A. A classification of response scale characteristics that affect data quality: a literature review. Qual Quant 2017; 52 (4): 1523-59.

24. Rojas-Barahona C, Zegers B, Förster C. La escala de autoestima de Rosenberg: Validación para Chile en una muestra de jóvenes adultos, adultos y adultos mayores. Rev med Chil 2009; 137 (6): 791-800.

25. Batista-Foguet J, Coenders G, Alonso J. Análisis factorial confirmatorio. Su utilidad en la validación de cuestionarios relacionados con la salud. Med Clin (Barc) 2004; 122 (1): 21-7.

26. Flora DB, LaBrish C, Chalmers RP. Old and new ideas for data screening and assumption testing for exploratory and confirmatory factor analysis. Front Psychol 2012; 3 (55): 1-21.

27. Muthén L, Muthén B. Mplus User's Guide. Los Angeles, US: Muthén \& Muthén; 1998-2012.

28. Brown TA. Confirmatory factor analysis for applied research. New York, US: The Guilford Press; 2006.

29. Bandalos DL, Finney SJ. Factor Analysis: Exploratory and Confirmatory. En: Hancock GR, Mueller RO, Edi- 
tores, Reviewer's guide to quantitative methods. New York, US: Routledge; 2010. p. 93-114.

30. Chen F. Sensitivity of goodness of fit indexes to lack of measurement invariance. Struct Equ Modeling 2007; 14 (3): 464-504.

31. Viladrich, C, Angulo-Brunet A, Doval E. A journey around alpha and omega to estimate internal consistency reliability. Anales de Psicología / Annals of Psychology 2017; 33 (3): 755-82.

32. Pallant J. SPSS survival manual: A step by step guide to data analysis using the SPSS. Crows Nest, Australia: Allen \& Unwin; 2011.

33. McDonald RP, Ho MH. Principles and practice in reporting structural equation analyses. Psychol Methods 2002; 7 (1): 64-82.

34. Asparouhov T, Muthén B. SRMR in Mplus. Technical appendix. Los Angeles, US: Muthén \& Muthén; 2018.

35. Lazić M, Jovanović V, Gavrilov-Jerković V. The general self-efficacy scale: New evidence of structural validity, measurement invariance, and predictive properties in relationship to subjective well-being in Serbian samples. Curr Psychol 2018; 1-12.

36. Bueno-Pacheco A, Lima-Castro S, Peña-Contreras E, Cedillo-Quizhpe C, Aguilar-Sizer M. Adaptación al español de la escala de autoeficacia general para su uso en el contexto ecuatoriano. Revista Iberoamericana de Diagnóstico y Evaluación Psicologica 2018; 48 (3): 5-17.

37. Judge TA, Bono JE. Relationship of core self-evaluations traits-self-esteem, generalized self-efficacy, locus of control, and emotional stability-with job satisfaction and job performance: A meta-analysis. J Appl Psychol 2001; 86 (1): 80-92. 